\title{
Understanding Obsessive Compulsive Disorder and Management Options
}

\author{
NA Giasuddin ${ }^{1}$, MJ Hossain ${ }^{2}$
}

\begin{abstract}
:
Obsessive compulsive disorder is a common mental health problem. It is characterized by obsession and compulsion. Obsession can be defined as unwanted, intrusive, recurrent and persistent thoughts, images or impulses which are not voluntarily produced, but are experienced as events that invade a person's consciousness. Compulsion can be defined as repetitive and seemingly purposeful behavior that is performed according to certain rules or in a stereotyped fashion and is not an end in itself but is usually intended to prevent some event or situation. The obsessions or compulsions interfere significantly with the person's normal routine, occupational functioning, usual social activities, or relationships. Obsessive-compulsive disorder and several related disorders are now put together into separate chapter in Diagnostic and Statistical Manual of Mental Disorders, $5^{\text {th }}$ Edition (DSM-5). Literature search was performed with the key words "Obsessive Compulsive Disorder", "Obsession", "Compulsion", "Treatment of Obsessive-Compulsive Disorder". Representative and leading researches from last 25 years were included in the study. Modern concepts of OCD began to evolve in the nineteenth century. Obsessions, in which insight was preserved, were gradually distinguished from delusions, in which it was not. The core features of OCD are remarkably similar from one country to the next, but its manifestations may differ for reasons of culture and experience. OCD is typically a chronic disorder with a waxing and waning course. In classical psychoanalytic theory, OCD was considered a regression from the Oedepal phase to the anal psycho-sexual phase of development. Functional brain imaging studies have produced a model for pathophysiology of OCD which involves hyperactivity in certain subcortical and cortical regions. The idea that abnormality in serotonergic neurotransmission underlies OCD arose from the observation that clomipramine, which inhibits serotonin and norepinephrine reuptake, relieved symptoms, whereas noradrenergic reuptake inhibitors did not. During the last 40 years there has been considerable progress in the pharmacological management of OCD. Behavioral therapy is also considered as an effective way of controlling OCD. Cognitive Behavioral Therapy (CBT) is a well-documented intervention for children, adolescents, and adults with OCD. Other biological approaches for obsessive-compulsive disorder include neurosurgery, deepbrain stimulation, electroconvulsive therapy, and repetitive transcranial magnetic stimulation. The neurosurgical techniques of cingulotomy and capsulotomy may provide clinical improvement among some patients with treatmentrefractory obsessive-compulsive disorder. Overall, stereotactic surgery should be viewed as a last option in treating refractory obsessive-compulsive disorder.
\end{abstract}

Key words: Obsessive-compulsive disorder, Deep-brain stimulation.

\section{Introduction:}

Obsessive compulsive disorder (OCD) is a common mental health problem. It is characterized by obsession and compulsion. Obsession can be defined as unwanted, intrusive, recurrent and persistent thoughts, images or impulses which are not voluntarily produced, but are experienced as events that invade a person's

\footnotetext{
1. Dr. Noor Ahmed Giasuddin, MBBS, M Phil (Psychiatry), Assistant Professor, Department of Psychiatry, Faridpur Medical College, Faridpur.
}

2. Dr. Md. Jahangir Hossain, MBBS, M Phil (Psychiatry), Associate Professor, Department of Psychiatry, Monno Medical College, Manikgonj.

Address of correspondence :

Dr. Noor Ahmed Giasuddin, MBBS, M Phil (Psychiatry), Assistant Professor, Department of Psychiatry, Faridpur Medical College, Faridpur. Phone:+88-01713682456, E-mail: sadidhk@gmail.com consciousness. Compulsion can be defined as repetitive and seemingly purposeful behavior that is performed according to certain rules or in a stereotyped fashion and is not an end in itself but is usually intended to prevent some event or situation ${ }^{1}$. The symptoms of OCD are accompanied by varying degrees of anxiety, depression, and depersonalization ${ }^{2}$. The obsessions or compulsions are time-consuming and interfere significantly with the person's normal routine, occupational functioning, usual social activities, or relationships ${ }^{3}$.

In Diagnostic and Statistical Manual of Mental Disorders, $4^{\text {th }}$ Edition (DSM-IV) ${ }^{4}$, obsessional disorders were classified as a type of anxiety disorder, but as recent studies have shown that obsessive-compulsive 
disorder involves distinct neuro-circuits, it and several related disorders are now put together into separate chapter in Diagnostic and Statistical Manual of Mental Disorders, $5^{\text {th }}$ Edition (DSM-5) ${ }^{5}$. Pharmacological and psychological treatments are both considered as an effective way of reducing the sign-symptoms of OCD. During the last forty years, there has been significant improvement in drug treatment of OCD. Currently OCD respond well to serotonin re-uptake inhibitors.

Modern concepts of OCD began to evolve in the nineteenth century. Obsessions, in which insight was preserved, were gradually distinguished from delusions, in which it was not. Compulsions were distinguished from "impulsions," which included various forms of paroxysmal, stereotyped and irresistible behavior. In his 1838 psychiatric textbook, Jean-Étienne Dominique Esquirol (1772-1840) described OCD as a form of monomania, or partial insanity. After 1850 s, French psychiatrists attempted to understand obsessions and compulsions within various broad categories. Henri Dagonet (1823-1902) considered compulsions to be a kind of impulsion and OCD a form of folie impulsive (impulsive insanity). Bénédict Augustin Morel (1809-1873) placed OCD within the category, "delire emotif" (diseases of the emotions). Valentin Magnan (1835-1916) considered OCD a "folie des degeneres" (psychosis of degeneration) ${ }^{7}$.

In 1877, German psychiatrist Karl Friedrich Otto Westphal ascribed obsessions to disordered intellectual function. Westphal's use of the term Zwangsvorstellung (compelled presentation or idea) gave rise to current terminology. In Great Britain Zwangsvorstellung was translated as "obsession," while in the United States it become "compulsion." The term "obsessive-compulsive disorder" emerged as a compromise.

As the twentieth century opened, both Pierre Janet (1859-1947) and Sigmund Freud (1856-1939) separated OCD from neurasthenia (inadequate tone of nerves). In Freud's view, the patient's mind responded maladaptively to conflicts between unacceptable, unconscious sexual or aggressive id impulses and the demands of conscience and reality. It regressed to concerns with control and to modes of thinking characteristic of the anal-sadistic stage of psychosexual development: ambivalence, which produced doubting, and magical thinking, which produced superstitious compulsive acts ${ }^{7}$.

\section{The nature of obsessions and compulsions:}

Obsessions are intrusive ideas, images, or impulses that patients see as illogical and uncontrollable. Obsessions are viewed as irrational by the individual whereas delusions are believed true by the individual. The most frequent obsessions involve thoughts about harm or danger to the self (e.g., fear of contamination), intense feelings of doubt, aggressive or sexual impulses, or somatic concerns.

Compulsions are repetitive behaviors or rituals that the individual feels compelled to carry out. They may be carried out to diminish feelings of anxiety and discomfort resulting from obsessive thoughts. Despite the immediate anxiety-reducing properties of the rituals, carrying out these behaviors only stave off anxiety for the short term. As the anxiety resurfaces, the ritual must be repeated. It is this repetitive cycle that perpetuates the behavior. No matter how many times the ritual is repeated, the anxiety remains and the neutralizing behaviors therefore must go on as well.

A compulsive washing ritual may address an individual's fears concerning contamination, whereas excessive checking may address concerns about safety. Avoidance may calm fears of the individual acting on aggressive or sexual obsessions. Other commonly observed compulsions include hoarding, counting, or repetitive confessions of perceived transgressions. Mental compulsions include thinking of special words or images to neutralize a thought, saying special prayers in a set manner, counting, constructing of mental lists, or mental reviewing.

OCD in children and adolescents is quite similar to OCD in adults. For example, the types of OCD symptoms (washing, checking, arranging, doubting, etc.) and the relative frequency of these symptoms are very consistent across the age range. Children, on the other hand, are typically unable to describe specific fears, often reporting that they don't know why they perform their rituals.

Cognitive decline can exacerbate or mimic symptoms of $\mathrm{OCD}^{8}$ and medical difficulties that are more prevalent in the elderly, such as cerebrovascular accidents, have been noted to produce OCD symptoms in previously healthy patients ${ }^{9}$. Patients with OCD frequently present with medical complaints. For example, fears of contamination may lead to tests for AIDS or other infectious diseases. Excessive washing may result in eczematous type reactions. Bowel obsessions such as fear of incontinence may result in repeated work-ups. Patients may watch what they eat very carefully or carry out procedures to make sure they are free of feces. Gum lesions may result from obsessive brushing of teeth.

\section{Cross cultural experience:}

OCD showed a prevalence of $2 \%-3 \%$ in five communities in the United States during the $1980 \mathrm{~s}^{10}$. 
This indicated that OCD was not a rare disorder. The cross-national collaborative study in community samples in Canada, the United Kingdom, Puerto Rico, Germany, Taiwan, Korea, and the Netherlands suggested a prevalence of $1.9 \%-2.5 \%{ }^{11}$, to $1 \%-2 \%$ in later studies by Nelson and Rice ${ }^{12}$ and Stein et al ${ }^{13}$. Research on children and adolescents reported prevalence ranges of $2 \%-3.6 \%{ }^{14}$.

Cross cultural experience indicates that the core features of OCD are remarkably similar from one country to the next, but its manifestations may differ for reasons of culture and experience ${ }^{15}$. The same morbid themes are found in Western and non-Western cultures. The morbid themes embedded in the religious obsessions and compulsions were familiar: dirt, orderliness, aggression, sex, washing, checking, repeating and slowness.

\section{Course and co-morbidity of OCD:}

OCD is typically a chronic disorder with a waxing and waning course. With effective treatment, the severity of symptoms can be reduced, but typically some symptoms remain ${ }^{16}$. Rasmussen and Eisen found that the most common comorbid condition in patients with OCD was major depression, occurring in approximately two thirds of patients. Prevalence of specific phobia $(22 \%)$, social phobia $(18 \%)$, and panic disorder $(12 \%)$ were also high ${ }^{17}$.

At National Institute of Mental Health, Dhaka it was found that among the 110 OCD cases the co-morbid disorders were depressive disorder (18.17\%), schizophrenia $(4.53 \%)$, substance related disorder $(0.9 \%)^{18}$. Uddin et $\mathrm{al}^{19}$ at the same institute found that $70 \%$ of the OCD patients had anxiety in clinical level and $66 \%$ had clinical depression; $30 \%$ of the sample was found to have strong suicidal risk.

\section{Etiology of OCD:}

Psychological models: In classical psychoanalytic theory, OCD was considered a regression from the Oedepal phase to the anal psycho-sexual phase of development. According to learning theorists, obsessions are conditioned stimuli and compulsions are established when a person develops active avoidance strategies in the form of ritualistic behaviors to control anxiety $^{3}$.

In one study $29 \%$ patients felt that an environmental precipitant had triggered their illness; most frequently increased responsibility, such as the birth of a child; or significant losses, such as a death in the family ${ }^{17}$. In case of women, $62 \%$ reported premenstrual worsening ${ }^{20}$.

\section{Biological Models:}

Functional Neuro anatomy: Functional brain imaging studies have produced a model for pathophysiology of OCD which involves hyperactivity in certain subcortical and cortical regions. It has been proposed that increased activity in the head of the caudate nucleus inhibits globus pallidus fibers that ordinarily dampen thalamic activity. The resulting increase in thalamic activity produces increased activity in orbitofrontal cortex, which, via the cingulate gyrus, completes the circuit to the caudate and produces increased activity in the head of the caudate. This hypothesis is supported by evidence from Magnetic Resonance Imaging (MRI) studies, which have found an abnormally small caudate in some OCD patients, and by Positron Emission Tomography (PET scan) studies, which have found increased metabolism in orbital frontal cortex, cingulate gyrus, and caudate, with decreases following successful treatment ${ }^{7}$. The results of neurosurgical treatment of OCD strongly support this hypothesis. Surgical interruption of this loop by means of cingulotomy, anterior capsulotomy or sub caudate tractotomy brings about symptomatic improvement in a large proportion of patients unresponsive to all other treatments. Cingulotomy interrupts this loop at the anterior cingulate cortex, thereby disrupting frontal cortical input into the Papez circuit and limbic system, which are believed to mediate anxiety and other emotional symptoms. Anterior capsulotomy (lesions within the anterior limb of the internal capsules) and sub caudate tractotomy (lesions in the substantia innominata, just under the head of the caudate nucleus) interrupt fronto-thalamic fibers, which may mediate the obsessive and compulsive components of OCD.

Neuro-imaging studies have documented consistent differences in regional brain activity between patients with OCD and control subjects, and the abnormal activity in patients with OCD shifts toward normal after either successful treatment with serotonin-reuptake inhibitors or effective behavioral therapy ${ }^{17}$.

Serotonin and Other Neurotransmitters: The hypothesis that an abnormality in serotonergic neurotransmission underlies OCD arose from the observation that clomipramine, which inhibits serotonin and norepinephrine reuptake, relieved symptoms, whereas noradrenergic reuptake inhibitors did not. The unique efficacy of clomipramine and the SSRIs remains the strongest support for this hypothesis. In patients with co-morbid Tourette's syndrome, tics and schizotypal personality disorder, treatment studies indicate a role for dopaminergic neurons. 
Genetic Contributions: The disorder has a significant genetic component and family studies have found 35\% of $1 \mathrm{st}$ degree relatives of OCD patients are also afflicted with the disorder. Twin studies and family studies strongly suggest that OCD can be inherited ${ }^{3}$.

\section{Impact on quality of life:}

Obsessive-compulsive disorder has a major impact on quality of life in several domains, including social functioning, employment, marriage and family relationships, and socioeconomic status. More severe the symptoms the poorer the quality of life, in the domain of social functioning ${ }^{21,22}$.

\section{Available treatments:}

\section{Pharmacological treatment:}

Current medications used for the treatment of OCD include antidepressant medications like Clomipramine, Sertraline, Fluvoxamine, Paroxetine, and Fluoxetine ${ }^{23}$. Clomipramine was the first agent which showed efficacy in $\mathrm{OCD}^{17}$. Clomipramine and Fluvoxamine showed better efficacy than other agents ${ }^{17}$. The initial response to pharmacotherapy is not evident until 4-6 weeks of treatment have elapsed and patients continue to improve for up to 3-4 months. Treatment after full remission of the symptoms should be continued long enough so that the neuronal dysfunction is fully treated before the medication is discontinued.

In contrast to cognitive-behavioral therapy, after which less than 25 percent of patients have a relapse, the discontinuation of serotonin-reuptake inhibitors results in a high rate of relapse. Total $89 \%$ of subjects who had clomipramine substituted with placebo during the maintenance phase of treatment, relapsed within 7 weeks, and the majority of patients on fluoxetine maintenance treatment relapsed within 12 weeks of discontinuing medication 24-26. These studies suggest that long-term maintenance treatment is required in OCD for most patients.

\section{Psychological treatments:}

Traditional psychotherapy, which sought to help the individuals gain insight or awareness into their problems, has proven to be ineffective in treating OCD. Relaxation techniques alone are not helpful in the treatment of OCD and are often used as a control form of therapy in studies. However, a specific type of therapy behavior that uses an "exposure and response prevention" approach has been more promising.

Behavior Therapy (BT) for OCD most specifically involves exposure $(\mathrm{E})$ and response or ritual prevention
(RP). Exposure capitalizes on the fact that anxiety usually attenuates after sufficient duration of contact with a feared stimulus. Repeated exposure is associated with decreased anxiety until, after multiple trials, the patient no longer fears contact with the specifically targeted stimulus. In order to achieve adequate exposure, it is usually necessary to help the patient block the rituals or avoidance behaviors, a process termed response or ritual prevention.

Cognitive Therapy (CT) for OCD involves providing clients with specific responses to the spikes. The educational component to $\mathrm{CT}$ involves helping sufferers understand that the contents of their OCD concerns are separate from and do not reflect their basic human nature or character. Providing reassurances and educating the OCD sufferer about how unlikely their risks actually are can be counterproductive and alienating. The immediate goal of therapy is not to eliminate the spikes or to feel better. The techniques are designed to manage anxiety and to stop the endless cycle of ritualizing. Brain imaging studies may predict response to behavioral therapy as well as pharmacotherapy.

Cognitive Behavioral Therapy (CBT) is a welldocumented intervention for children, adolescents, and adults with OCD. In milder OCD and in younger patients, CBT alone may be the initial choice. As severity increases, medications alone or with CBT is used as the beneficial treatment ${ }^{17}$.

\section{Other non-pharmacological strategies:}

Other biological approaches for obsessive-compulsive disorder include neurosurgery, deep-brain stimulation, electroconvulsive therapy, and repetitive transcranial magnetic stimulation ${ }^{27}$. The neurosurgical techniques of cingulotomy and capsulotomy may provide clinical improvement among some patients with treatmentrefractory obsessive-compulsive disorder. Overall, stereotactic surgery should be viewed as a last option in treating refractory obsessive-compulsive disorder.

\section{References:}

1. De Silva P, Rachman S. Obsessive--compulsive disorder: the facts. $2^{\text {nd }}$ ed. Oxford; New York: Oxford University Press; 1998.p.141. (Oxford medical publications)

2. Harrison PJ, Cowen P, Burns T, Fazel M. Shorter Oxford textbook of psychiatry. Seventh edition. Oxford: Oxford University Press; 2018.p.884

3. Sadock BJ, Sadock VA, Ruiz P. Kaplan \& Sadock's synopsis of psychiatry: behavioral sciences/clinical psychiatry. Eleventh edition. Philadelphia: Wolters Kluwer; 2015.p.1472. 
4. American Psychiatric Association, editors. Diagnostic and statistical manual of mental disorders: DSM-IV-TR. 4th ed., text revision. Washington, DC: American Psychiatric Association; 2000.p.943.

5. American Psychiatric Association, editors. Diagnostic and statistical manual of mental disorders: DSM-5. 5th ed. Washington, D.C: American Psychiatric Association; 2013.p.947.

6. Telles-Correia D, Marques JG. Melancholia before the twentieth century: fear and sorrow or partial insanity? Front Psychol. 2015; $6: 81$.

7. History [Internet]. Obsessive-Compulsive and Related Disorders. [cited 2020 Mar 28]. Available from: http://med.stanford.edu/ ocd/treatment/history.html

8. Markovitz PJ. Treatment of anxiety in the elderly. J Clin Psychiatry. 1993 May; 54 Suppl:64-8.

9. Simpson S, Baldwin B. Neuropsychiatry and SPECT of an acute obsessive-compulsive syndrome patient. Br J Psychiatry J Ment Sci. 1995 Mar; 166(3):390-92.

10. Karno M, Golding JM, Sorenson SB, Burnam MA. The epidemiology of obsessive-compulsive disorder in five US communities. Arch Gen Psychiatry. 1988 Dec; 5(12):1094-99.

11. Weissman MM, Bland RC, Canino GJ, Greenwald S, Hwu HG, Lee $\mathrm{CK}$, et al. The cross national epidemiology of obsessive compulsive disorder. The Cross National Collaborative Group. J Clin Psychiatry. 1994 Mar; 55 Suppl:5-10.

12. Nelson E, Rice J. Stability of diagnosis of obsessive-compulsive disorder in the Epidemiologic Catchment Area study. Am J Psychiatry. 1997 Jun; 154(6):826-31

13. Stein MB, Forde DR, Anderson G, Walker JR. Obsessivecompulsive disorder in the community: an epidemiologic survey with clinical reappraisal. Am J Psychiatry. 1997 Aug; 154(8):1120-26.

14. Flament MF, Whitaker A, Rapoport JL, Davies M, Berg CZ, Kalikow K, et al. Obsessive compulsive disorder in adolescence: an epidemiological study. J Am Acad Child Adolesc Psychiatry. 1988 Nov; 27(6):764-71

15. Stein DJ, Rapoport JL. Cross-Cultural Studies and ObsessiveCompulsive Disorder. CNS Spectr. 1996 Sep; 1(1):42-6.

16. Caulfield C. Obsessive-Compulsive Disorder Edited By Mario Maj, Norman Sartorius, Ahmed Okasha \&amp; Joseph Zohar. Chichester: John Wiley \&amp; Sons. 2000. 308 pp. $\$ 60.00$ (hb). ISBN 047187163 X. Br J Psychiatry. 2002; 180(3):289-90.

17. Rasmussen SA, Eisen JL. The epidemiology and clinical features of obsessive compulsive disorder. Psychiatr Clin North Am. 1992 Dec; 15(4):743-58

18. Firoz A, Rahman A, Alam M, Algin S, Karim M, Rahman S. Profile of OCD patients. Bangladesh J Psychiatry. 2004; 18(2):5-10.

19. Uddin M, Mohit M, Chowdhury M, Hossain M, Hossain M. Anxiety, Depression, hopelessness and obsessive-compulsive subtypes among 50 clients having obsessive-compulsive disorder. In Dhaka, Bangladesh; 2006.

20. Williams KE, Koran LM. Obsessive-compulsive disorder in pregnancy, the puerperium, and the premenstruum. J Clin Psychiatry. 1997 Jul; 58(7):330-4; quiz 335-6.

21. Jenike MA, Baer L, Minichiello WE, editors. Obsessivecompulsive disorders: practical management. $3^{\text {rd }}$ ed. St. Louis, MO: Mosby; 1998.p.885.
22. Koran LM. Quality of life in obsessive-compulsive disorder. Psychiatr Clin North Am. 2000 Sep; 23(3):509-17.

23. Cartwright C, Hollander E. SSRIs in the treatment of obsessivecompulsive disorder. Depress Anxiety. 1998; 8 Suppl 1:105-13.

24. Greist JH, Jefferson JW, Kobak KA, Katzelnick DJ, Serlin RC. Efficacy and tolerability of serotonin transport inhibitors in obsessive-compulsive disorder. A meta-analysis. Arch Gen Psychiatry. 1995 Jan; 52(1):53-60.

25. Piccinelli M, Pini S, Bellantuono C, Wilkinson G. Efficacy of drug treatment in obsessive-compulsive disorder. A meta-analytic review. Br J Psychiatry J Ment Sci. 1995 Apr; 166(4):424-43.

26. Pato MT, Zohar-Kadouch R, Zohar J, Murphy DL. Return of symptoms after discontinuation of clomipramine in patients with obsessive-compulsive disorder. Am J Psychiatry. 1988 Dec; 145(12):1521-25

27. Giasuddin NA, Nahar JS, Morshed NM, Balhara YPS, Sobhan MA. Efficacy of combination of fluoxetine and cognitive behavioral therapy and fluoxetine alone for the treatment of obsessive compulsive disorder. Pak J Pharm Sci. 2013 Jan; 26(1):95-8. 